\title{
Evaluation of renal perfusion by Doppler ultrasonography and the effect of Ulinastatin to it in a porcine model of septic shock
}

\section{Chen-Chen Hang}

Beijing Chao-Yang Hospital, Capital Medical University

\section{Yu-Hong Guo}

Beijing Hospital of Traditional Chinese Medicine, Capital Medical University

\section{Chun-Sheng Li}

Beijing Friendship Hospital, Capital Medical University

Shuo Wang ( $\square$ ysys2001@yeah.net )

Beijing Hospital of Traditional Chinese Medicine https://orcid.org/0000-0001-6830-0817

\section{Original research}

Keywords: Acute kidney injury, Septic shock, Doppler ultrasonography, Contrast enhanced ultrasound, Ulinastatin

Posted Date: March 30th, 2021

DOl: https://doi.org/10.21203/rs.3.rs-339073/v1

License: (9) (1) This work is licensed under a Creative Commons Attribution 4.0 International License. Read Full License 


\section{Abstract}

Objective To develop a reliable and noninvasive method to evaluate the renal perfusion in a porcine model of septic shock and investigate the effect of UTI on it. Methods Thirty-two healthy male domestic pigs were randomly assigned to one of four groups: a sham group ( $\mathrm{SH}, \mathrm{n}=5)$; a septic shock group (SS, $\mathrm{n}=9)$; a septic shock group treated with vancomycin $(15 \mathrm{mg} / \mathrm{kg})(V A N, \mathrm{n}=9)$ and a septic shock group treated with UTI $(50,000 \mathrm{U} / \mathrm{kg})$ + vancomycin $(\mathrm{UTI}, \mathrm{n}=9)$. Contrast-enhanced ultrasound (CEUS) of kidney at the baseline and the end of protocol $(24 \mathrm{~h})$ were performed. The spectrum of interlobar or arcuate artery was selected to calculate the corrected resistive index (cRI). Sulphur hexafluoride (SF6) microbubbles were bolus injected via venous catheter. The peak intensity $(\mathrm{Pi})$ and area under curve (AUC) were calculated by a time-intensity curve (TIC). Results cRI increased significantly at the end of the protocol except the $\mathrm{SH}$ groups, significant difference was found between each experimental group and the $\mathrm{SH}$ group. Linear regression was found between the cardiac output (CO) and $\mathrm{Pi}(\mathrm{Pi}=7.082 \times \mathrm{CO}+5.026$, $\mathrm{R} 2=0.752, \mathrm{~F}=84.878, \mathrm{P}<0.001)$. The AUC decreased significantly post-injury in the SS and VAN group. All above parameters were improved by the UTI treatment, significant differences were found between the UTI and SS or VAN group respectively $(P<0.05)$. Conclusions Septic shock occasionally accompanies with a significant low perfusion in renal microcirculation and UTI can improve it significantly.

\section{Introduction}

As a complex syndrome of multiple organ dysfunction induced by severe infection, sepsis and septic shock is one of the main causes of mortality in critically ill patients. Although treatment strategies have been improved continually, there are still remaining issues about the complications induced by pathophysiologic development, such as acute renal injury (AKI). AKI occurs in about $19 \%$ of patients with moderate sepsis, $23 \%$ of those with severe sepsis, and $51 \%$ of those with septic shock when blood cultures are positive [1]. The hemodynamic fluctuation of septic shock includes a phase of high cardiac output (CO) with low peripheral resistance followed by low $\mathrm{CO}$ with high peripheral resistance. The difference between the flow of kidney and other organs is the reaction to the vasoconstriction. It makes the difference of kidney to hemodynamic fluctuation with other organs, which is the decreasing perfusion when the patients suffered with sepsis [2].

Color Doppler flow imaging (CDFI) is a rapid, noninvasive, and repeatable technology for detecting AKI, which has been suggested for assessing changes of renal perfusion in critically ill patients $[3,4]$. The contrast-enhanced ultrasound (CEUS) allows noninvasive estimation of the renal blood flow more accurately and provides quantitative measurement of local blood volume and velocity in selective regions of interest (ROI). Ulinastatin (UTI), a human protease inhibitor isolated from male urine, is a glycoprotein with a typical Kuniz protease inhibitor structure that has a broad spectrum of enzyme inhibitory activity $[5,6]$. It has been used clinically for the prevention of multiple organ dysfunction [7, 8]. Its protecting effect to AKI has been a hotly debated topic in recent years and remains unclear. 
The goal of this study was to develop a reliable and noninvasive method to evaluate the renal perfusion in a porcine model of septic shock and investigate the effect of UTI on it.

\section{Materials And Methods}

\section{Animal preparation}

Thirty-two healthy male domestic pigs aged 8-10 weeks and weighing $30 \pm 2 \mathrm{~kg}$ were used for the study. Anesthesia was induced by midazolam $0.2 \mathrm{mg} / \mathrm{kg}$ injected intramuscularly and followed with continuous intravenous infusion of pentobarbital ( $8 \mathrm{mg} / \mathrm{kg} / \mathrm{h})$. Ringer's solution was administered intravenously to maintain sufficient preload. The right femoral artery and vein were dissected to insert a 5-Fr PiCCO catheter (Pulsiocath PV2015L20; Pulsion Medical Systems, Munich, Germany) into the descending aorta and a central venous catheter into the right atrium respectively. The arterial and central venous catheters were connected to a monitor (Philips Medical Systems; Best, Holland) for continuous monitoring the hemodynamics and blood temperature.

All animals were intubated using a cuffed 6.5-mm endotracheal tube. Mechanical ventilation was provided by a ventilator (Evita 4; Dräger Medizintechnik, Lübeck, Germany) with a tidal volume of 8 $\mathrm{mL} / \mathrm{kg}$, a respiratory frequency of 12 breaths/min and a positive end-expiratory pressure (PEEP) of $5 \mathrm{~cm}$ $\mathrm{H}_{2} \mathrm{O}$. The respiratory frequency and the fraction of inspired oxygen $\left(\mathrm{FiO}_{2}\right)$ were adjusted to maintain an end tidal concentration of carbon dioxide $\left(\mathrm{EtPCO}_{2}\right)$ of $35-40 \mathrm{mmHg}$ and an arterial oxygen saturation $\left(\mathrm{SpO}_{2}\right)$ of more than $90 \%$, respectively.

\section{Experimental protocols}

Baseline data were obtained after a recovery period of 1 hour. Animals were randomly assigned to one of four groups: a sham group ( $\mathrm{SH}, \mathrm{n}=5)$; a septic shock group (SS, $\mathrm{n}=9)$; a septic shock group treated with vancomycin (500mg, Eli Lilly, IN) (VAN, n=9) and a septic shock group treated with UTI $(50,000 \mathrm{U}$, Techpool, Guangdong, China) + vancomycin $(U T I, n=9)$. The number of samples was confirmed according to our previous investigation [9]. No other procedures were performed in the SH group apart from catheter insertion and mechanical ventilation. All animals of other three experimental groups were exposed to smoke from $50 \mathrm{~g}$ of burning cotton using a bee smoker according to previously described methods [10-12]. Arterial carboxyhemoglobin levels were measured using a portable monitor (Rad-57; Masimo Corp., Irvine, CA) to ensure that each animal had received an equivalent dose of smoke. After 12 breaths of cotton smoke were insufflated into the lungs, animals were allowed to breathe normal air for $2 \mathrm{~min}$. Four sets of smoke inhalations (total of 48 breaths) were performed.

Following smoking injury, $3 \times 10^{11}$ colony forming units (CFU) of live methicillin-resistant Staphylococcus aureus (MRSA) were instilled into the lungs of animals except sham group via a bronchoscope [9]. Afterwards, $15 \mathrm{mg} / \mathrm{kg}$ of vancomycin and $15 \mathrm{mg} / \mathrm{kg}$ of vancomycin $+50,000 \mathrm{U} / \mathrm{kg}$ of UTI in $100 \mathrm{~mL}$ saline were infused into the central venous catheter within 30 min every $12 \mathrm{~h}$ in the VAN and UTI group respectively. 
All animals were continuously monitored in the whole protocol for $24 \mathrm{~h}$. At the end of the protocol, $10 \mathrm{~mL}$ of blood were extracted into an aerobic culture bottle (BacT/ALERT FA; bioMérieux Inc., Durham, NC) for blood culture. The right kidney was dissected for histopathology test. The diagram of experiment was shown in Figure 1.

\section{Hemodynamics}

$10 \mathrm{~mL} 4^{\circ} \mathrm{C}$ saline were injected into the right atrium through the central venous catheter to determine $\mathrm{CO}$ by the thermodilution method. The systemic vascular resistance (SVR) was calculated by the monitor. Heart rate (HR) and mean arterial pressure (MAP) were recorded continuously.

Arterial blood samples for blood gas analysis and lactic acid (Lac) (GEM Premier 3000 Blood Gas Analyzer; Instrumentation Laboratory, Lexington, MA, USA) were collected at the baseline and $24 \mathrm{~h}$ after injury.

\section{CDFI and CEUS}

CDFI and CEUS were performed on right kidney at the baseline and the end of protocol (24h). All studies were performed by an experienced ultrasound physician, using an iU22 probe (Philips Ultrasound, Bothell, WA, USA). The probe was put on the right side of the animal's abdomen to obtain a clear image of the right kidney. An interlobar or arcuate artery was selected to obtain the result of measurement. The spectrum was considered optimal when at least three similar consecutive waveforms were visualized (Fig 2A-D). The corrected resistive index (cRI) was calculated using the following equations [13]. Three measurements were performed and averaged to obtain the mean $\mathrm{CRI}$ value.

$\mathrm{RI}=$ (peak systolic velocity - minimum diastolic velocity) / peak systolic velocity

$\mathrm{cRI}=($ observed RI $-0.0026 \times[80$ - observed HR])

For CEUS, $2 \mathrm{ml}$ of sulphur hexafluoride $\left(\mathrm{SF}_{6}\right)$ microbubbles (8ul SF $\left.6 / \mathrm{ml}\right)$ (SonoVue®, Bracco Suisse SA, Plan-Les-Ouates, Switzerland) were bolus injected via the central venous catheter. ROI (26mm²) was manually drawn within the renal cortex to create a time-intensity curve (TIC) with the ultrasound system's QLAB software. The peak intensity (Pi), area under curve (AUC), time from peak to one half (Th), time to peak (Tp) and wash in slope (Slope) were calculated by the software automatically (Fig $2 \mathrm{E}-\mathrm{H}$. For videos, see supplemental files 1-4.). Three injections were done with the intervals of $5 \mathrm{~min}$ to get an average value. The same renal cortex ROI to prevent the influence of the respiratory motion was used for each bolus injection.

\section{Pathologic examination and TUNEL assay}

After ROSC $24 \mathrm{~h}$, animals were sacrificed. The upper pole of right renal cortex was surgically removed and preserved in $4 \%$ paraformaldehyde or $2 \%$ glutaraldehyde to observe pathologic changes under a light microscope (LM) or transmission electron microscope (EM) (HT7700; Hitachi, Ibaraki Prefecture, Japan). 
Then, a grade of renal injury of semi-quantitative evaluation was done in each animal (Table 1). Terminal deoxynucleotidyl transferase mediated 2-deoxyuridine 5-triphosphate nick end labeling (TUNEL) assay was used to label cells that suffered severe DNA damage/fragmentation induced by apoptotic signaling cascades. The TUNEL positive cells were counted to determine the apoptotic index (Al). Al = apoptotic cells stained brown / total TUNEL positive cells. The pathologic evaluations were performed by an independent pathologist who has more than ten years of experience and was blinded to this study.

\section{Statistical analysis}

Statistical analysis was performed by SPSS 19.0 software. Discrete variables were compared with a Fisher's exact test. All continuous variables in this study were normal distribution, which were confirmed by the Kolmogorov-Smirnov test and reported as mean \pm SD. The time and treatment effects were detected by the repeated measure ANOVA. The difference between the groups was compared by the multivariate ANOVA. Linear regression was performed to determine the correlations of parameters. A value of $P<0.05$ was considered statistically significant.

\section{Results}

\section{Outcomes}

No significant difference in baseline blood temperature was found among the four groups $(F=0.110$, $\mathrm{P}=0.954$ ). There was a rapid increase in blood temperature to more than $40^{\circ} \mathrm{C}$ in the three experiment groups within $2 \mathrm{~h}$ post-injury, but it stayed normal in the $\mathrm{SH}$ group at the whole protocol. One animal in the SS and VAN group died at $16 \mathrm{~h}$ and $20 \mathrm{~h}$ post-injury respectively. All other animals remained alive until the end of the protocol. All blood cultures of the SH, VAN and UTI group were negative, whereas $6 / 9$ animals in the SS group were positive with MRSA.

\section{Hemodynamics and oxygenation}

All parameters in the SH group stayed at a relatively normal level throughout the entire protocol. The HR increased very significantly in the SS group, which was higher than $133 \pm 7$ beats/min at $2 \mathrm{~h}$ post-injury. The changes of HR in the VAN group were similar with the SS group. Whereas in the UTI group, HR decreased slowly after $12 \mathrm{~h}$ post-injury, significant differences were found compared with the SS and VAN group $(P<0.001$, Fig $3 A)$.

In the SS and VAN group, MAP decreased to $60-70 \mathrm{mmHg}$ at $2 \mathrm{~h}$ post-injury, and stayed low. MAP of the UTI group also decreased at $2 \mathrm{~h}$ post-injury, but improved progressively after that. Significant differences were found compared with the SS or VAN group at each time point starting at $6 \mathrm{~h}$ post-injury $(P<0.01$, Fig 3B). The changes of $\mathrm{CO}$ and SVR in the SS and VAN group were in opposite directions: the CO increased at $2 \mathrm{~h}$ post-injury and then decreased gradually (Fig $3 \mathrm{C}$ ), whereas the SVR decreased at the same time point and then increased gradually (Fig 3D). The fluctuation of $\mathrm{CO}$ in the UTI group was relieved. At $24 \mathrm{~h}$ post-injury, $\mathrm{CO}$ was improved and significant difference was found compared with the SS or VAN group 
respectively. SVR in the UTI groups increased slightly before $6 \mathrm{~h}$ post-injury and stayed stable at the level of slightly higher than the SH group after that. It was significantly lower than the SS or VAN group $(P<0.001)$.

The ratio of the partial pressure of arterial oxygen $\left(\mathrm{PaO}_{2}\right)$ to the $\mathrm{FiO}_{2}$ in the SS and VAN group decreased after cotton smoke-inhalation, and was lower than 200. $\mathrm{PaO}_{2} / \mathrm{FiO}_{2}$ in the UTI group was higher than the SS and VAN group $(P<0.001)$, even was lower than the SH group $(P<0.001)$ (Fig $4 A)$. Lactic acid $(\mathrm{Lac})$ levels significantly increased in all experimental groups than the $\mathrm{SH}$ group $(P<0.001$, Fig 4B), whereas it was lower in the UTI group than the SS and VAN group $(P<0.001)$.

\section{CDFI and CEUS}

No significant difference of CRI was found at the baselines in all groups, but increased significantly at the end of the protocol except the SH groups, significant difference was found between each experimental group and the $\mathrm{SH}$ group $(\mathrm{P}<0.001$, Fig $5 \mathrm{~A})$.

Pi decreased significantly after injury in all experimental groups, but was higher in the UTI group than the SS and VAN group $(P<0.001$, Fig $5 B)$. Linear regression was found between the $C O$ and $P i(P i=7.082 \times$ $\left.\mathrm{CO}+5.026, \mathrm{R}^{2}=0.752, \mathrm{~F}=84.878, \mathrm{P}<0.001, \mathrm{Fig} 6\right)$. Since there was similar regression coefficient in each group, we used $\mathrm{CO}$ as a covariate to do the analysis again. Interestingly, the significant differences of $\mathrm{Pi}$ among the groups disappeared.

The AUC also decreased post-injury in the SS and VAN group, but was improved in the UTI group. Significant difference was found between the UTI and SS or VAN group, but was not found between the $\mathrm{SH}$ and UTI group ( $\mathrm{P}<0.001$, Fig $5 \mathrm{C})$.

Th and Tp increased significantly in the SS and VAN group, whereas the Slope decreased significantly. All above parameters were improved by the UTI treatment, significant differences were found between the UTI and SS or VAN group respectively $(P<0.05$, Fig 5D-F).

\section{Renal histopathology}

Compared with the SH group, the renal injury was prominent in all experimental groups (Fig 7A-D), especially in the mesenchyme. Glomerular capillaries hyperemia, fibroblasts proliferation near the glomerulus, micro-thrombus formation and inflammatory cells diffusive infiltration in the mesenchyme were observed. Under the LM, there was not significant injury in the SH group, but the grades of renal injury was higher in the three experimental groups; whereas it was lower in the UTI group than the SS and VAN group $(P<0.001$, Table 2). Under the EM in the SS group, the chromatin and nucleus vacuolar of epithelium were degenerated; the mitochondria swelled, and the cristae disappeared (Fig 8A, B).

TUNEL assay revealed that there were greater numbers of apoptotic cells in the SS and VAN group than the SH and UTI group. Significant difference was found between the UTI and SS or VAN group (Table 2, Fig 7E-H). 


\section{Discussion}

An estimated 5-20\% of critically ill patients experience an episode of AKI during the course of illness in many cases accompanied by multiple organ dysfunction syndromes [14-16]. A recent analysis from the PICARD Study Group observed a changing range of AKI in critically ill patients, characterized by a large burden of comorbidity, even progressed to pre-existing chronic kidney disease and extensive extra renal complications necessitating dialysis [17]. Approximately $4.9 \%$ of admissions to intensive care units required renal replacement therapy [18]. A prospective hospital-based study in the UK found that the incidence of renal replacement therapy for AKI was 131 per million per year [19].

After the sufficient fluid resuscitation, it is possible that the renal perfusion can recovery, but the microcirculation in the renal cortex cannot recovery to normal $[20,21]$. Hypovolaemia leading to a fall in systemic blood pressure activates the neurohumoral vasoconstrictive system to maintain the blood pressure and the cerebral perfusion but not the kidney [22]. The autoregulating of kidney to volume makes its perfusion stable within fairly narrow limits. When the MAP $>80 \mathrm{mmHg}$, the autoregulation can make the glomerular filtration rate (GFR) normal with the perfusion; but when MAP $<80 \mathrm{mmHg}$, the GFR decreases relating with the low perfusion $[23,24]$. In our model, MAP and the opposite changes of $C O$ and SVR followed the hemodynamic features of septic shock. Our previous investigation also confirmed the hemodynamic features of this model [9]. The MAP of all experiment groups decreased below $80 \mathrm{mmHg}$ post-injury, which could certainly make the renal perfusion decrease.

Renal Doppler ultrasonography is a noninvasive method to evaluate renal vascular condition. RI is a useful index to evaluate renal vascular resistance, which holds promise in monitoring renal function and in predicting AKI of critically ill patients [25]. We calculated cRI to correct the influence of heart rate, and found an increase of it in our model, which indicated the vasoconstriction of kidney. Whereas we did not find any change of cRI after UTI treatment, which demonstrated that UTI could not change the tension of renal vascular, but did not mean it was useless in regulating the renal microcirculation.

Microbubbles used in CEUS remain strictly intravascular, with no interstitial diffusion or urine excretion. They produce more harmonic signal than surrounding tissue, and are very sensitive to the echo sound prober. Because of the increased sensitivity of harmonic imaging, tissue perfusion on a capillary level can be detected using these media. Therefore, they can be viewed as blood pool markers enabling functional vascular imaging [26]. We used TIC derived from CEUS to detect the blood perfusion during septic shock and found that the AUC decreased significantly. AUC was a parameter related with the blood volume of the kidney. In the UTI group, it was significantly increased by the drug treatment. There was no significant difference compared with the SH group.

$\mathrm{Pi}$ is another parameter related with the renal perfusion. In theory, if the same dose of microbubbles were bolus infused into the circulation and detected by the prober on kidney, there would be a same Pi in each group. If $\mathrm{CO}$ decreased, only the coming time of Pi would be delayed because of the low perfusion but without the strength decreasing. In another word, there would be an increased Tp with normal Pi. Whereas if $\mathrm{CO}$ were too low, before the whole dose of microbubbles filled with the kidney, the washing out process 
have already begun with flow, which might decrease Pi. In our investigation, as we expected, Tp increased and Pi decreased significantly in the SS and VAN group. We did the regression analysis between the CO and $\mathrm{Pi}$ and found that there were similar regression coefficients in the four groups. Since there was lower $\mathrm{CO}$ in the SS and VAN groups, it might decrease Pi. So when CO was put into the ANOVA model as a covariate, the difference among the groups disappeared, which confirmed that a lower CO might decrease $\mathrm{Pi}$. In the UTI group, both of Pi and Tp were improved. Significant differences were found compared with the SS and VAN group.

Th and Slope are the parameters related with the wash in speed of microbubbles. In septic shock, both of them deteriorated with the poor perfusion. Th increased and Slope decreased, whereas they were improved by the UTI treatment. Unlike the parameter relating with the vascular tension, such as cRI, the parameters of perfusion, such as AUC, $\mathrm{Pi}$, Th and Slope, were improved by UTI, which demonstrated that the protection effect of UTI was not by the change of vascular resistance, but by the improvement of flow.

Oxygenation $\left(\mathrm{PaO}_{2} / \mathrm{FiO}_{2}\right)$ was also improved by the UTI treatment, together with the improved renal perfusion, the global aerobic metabolism is better. It was not surprise that the Lac decreased.

The histopathology confirmed the injury of kidney by septic shock and the improvement by UTI treatment. Previous investigation showed that $35-50 \%$ of cases of acute tubular necrosis could be attributed to sepsis [27, 28]. In our model, there was not an obvious tubular necrosis observed, which might because of the relative short duration of the protocol, but significant injures in the interstitium were found in the SS and VAN group under LM. These injuries might progress to tubular necrosis after a relative long time. Under EM, the injuries of cells were the mitochondria swelling and the chromatin degenerating that contributed to apoptosis. Investigation has found the mechanisms of mitochondrial dysfunction in the hyper metabolism organs, such as kidney [29]. The TUNEL analysis also confirmed the apoptosis of the cells. The histopathologic injury was ameliorated by UTI treatment, which may be due to the improvement of the renal perfusion.

CEUS is a reliable method to evaluate the renal perfusion, however the relationship between true renal blood flow and ultrasonic estimation's is complex and depends on many parameters, such as the microbubble concentration, region of interest position, complex distribution of the acoustic energy in the imaging plane. The patient respiration or other gross movements, regions that were not previously insonified by the stationary transducer can also influence the accurate of test, so the experience of the operators was very important to have the accurate results.

As a typical Kuniz protease inhibitor, study has demonstrated that UTI can regulate many cytokines' expression by NF-KB pathway [30]. Blood flow to the kidney is pronounced as the kidneys receive an estimated $25 \%$ of the total abdominal aorta blood flow and filter $120-150 \mathrm{ml}$ plasma per minute, which make it expose to the first strike of the cytokines. We did not test the cytokines in this investigation for further expounding the mechanism. Intensive studies should be done following this one. 


\section{Conclusions}

AKI, which occasionally occurs in septic shock, accompanies with a significant low perfusion in renal microcirculation. CEUS is a reliable method to evaluate the flow of kidney and UTI can improve the renal perfusion significantly.

\section{Abbreviations}

AKI: acute renal injury, CO: cardiac output, CDFl: Color Doppler flow imaging, CEUS: contrast-enhanced ultrasound, ROI: regions of interest, UTI: Ulinastatin, PEEP: positive end-expiratory pressure, EtPCO2: end tidal concentration of carbon dioxide, SpO2: arterial oxygen saturation, $\mathrm{CFU}$ : colony forming units, MRSA: methicillin-resistant Staphylococcus aureus, SVR: systemic vascular resistance, HR: heart rate, MAP: mean arterial pressure, Lac: lactic acid, cRl: corrected resistive index, SF6: sulphur hexafluoride, TIC: timeintensity curve, Pi: peak intensity, AUC: area under curve, Th: time from peak to one half, LM: light microscope, EM: transmission electron microscope, TUNEL: terminal deoxynucleotidyl transferase mediated 2-deoxyuridine 5-triphosphate nick end labeling, Al: apoptotic index

\section{Declarations}

\section{Ethics approval and consent to participate}

This study was approved by the Animal Care and Use Committee of Beijing Chao-Yang Hospital, Capital Medical University (No. SCXK 11-00-002), and the use of all animals that received treatment was in compliance with the National Research Council's 1996 Guide for the Care and Use of Laboratory Animals.

\section{Consent for publication}

Not applicable

\section{Availability of data and material}

All data generated or analyzed during this study are included in this published article.

\section{Competing interests}

The authors declare that they have no competing interests.

\section{Funding}

The Planning Project of Beijing Municipal Administration of Traditional Chinese Medicine (JJ2018-52).

\section{Authors' contributions}


HCC: operating and manuscript writing. GYH: operating, data acquisition and statistical analysis. LCS: final approval of the manuscript. WS: conception, design and final approval of the manuscript. All authors read and approved the final manuscript.

\section{Acknowledgements}

Not applicable

\section{Conflicts of Interests}

None.

\section{Funding Support:}

The Planning Project of Beijing Municipal Administration of Traditional Chinese Medicine (JJ2018-52)

\section{References}

1. Schrier RW, Wang W. Acute renal failure and sepsis. N Engl J Med. 2004;351:159-69.

2. Boffa JJ, Trendshorst WJ. Maintenance of renal vascular reactivity contributes to acute renal failure during endotoxemic shock. J Am Soc Nephrol. 2005;16(1):117-24.

3. Schnell D, Darmon M. Renal Doppler to assess renal perfusion in the critically ill: a reappraisal. Intensive Care Med. 2012;38:1751-60. PMID: 23001447.

4. Schnell D, Camous L, Guyomarc'h S, Duranteau J, Canet E, Gery P, et al. Renal perfusion assessment by renal Doppler during fluid challenge in sepsis. Crit Care Med. 2013;41:1214-20.

5. Puqia MJ, Valdes R Jr, Jortani SA. Bikunin (urinary trypsin inhibitor): structure, biological relevance, and measurement. Adv Clin Chem. 2007;44:223-45.

6. Inoue $\mathrm{K}$, Takano $\mathrm{H}$, Yanagisawa $\mathrm{R}$, et al. Protective role of urinary trypsin inhibitor in acute lung injury induced by lipopolysaccharide. Exp Biol Med (Maywood). 2005;230:281-7.

7. Yang Q, Liu X, Liu M, et al. Ulinastatin-mediated protection against zymosan-induced multiple organ dysfunction in rats. Biologicals. 2010;38:552-6.

8. Ogawa M, Nishibe S, Mori T, et al. Effect of human urinary trypsin inhibitor on granulocyte elastase activity. Res Commun Chem Pathol Pharmacol. 1987;55:271-4.

9. Wang S, Wang JY, Wang T, et al. A novel porcine model of septic shock induced by acute respiratory distress syndrome due to methicillin-resistant staphylococcus aureus. Chin Med J. 2017;130:122635 .

10. Murakami K, Bjertnaes LJ, Schmalstieg FC, et al. A novel animal model of sepsis after acute lung injury in sheep. Crit Care Med. 2002;30:2083-90.

11. Enkhbaatar P, Joncam C, Traber $L$, et al. Novel ovine model of methicillin-resistant Staphylococcus aureus-induced pneumonia and sepsis. Shock. 2008;29:642-9. 
12. Jonkam CC, Bansal K, Traber DL, et al. Pulmonary vascular permeability changes in an ovine model of methicillin-resistant Staphylococcus aureus sepsis. Crit Care. 2009;13:R19.

13. Schnell D, Darmon M. Renal Doppler to assess renal perfusion in the critically ill: A reappraisal. Intensive Care Med. 2012;38:1751-60.

14. Brivet FG, Kleinknecht DJ, Loirat P, Landais PJ. Acute renal failure in intensive care units: causes, outcome, and prognostic factors of hospital mortality: a prospective, multicenter study. Crit Care Med. 1996;24:192-98.

15. de Mendonca A, Vincent JL, Suter PM, et al. Acute renal failure in the ICU: risk factors and outcome evaluated by the SOFA score. Intensive Care Med. 2000;26:915-21.

16. Liano F, Junco E, Pascual J, Madero R, Verde E. The spectrum of acute renal failure in the intensive care unit compared with that seen in other settings. Kidney Int Suppl. 1998;66:16-24.

17. Mehta RL, Pascual MT, Soroko S, et al. Spectrum of acute renal failure in the intensive care unit: the PICARD experience. Kidney Int. 2004;66:1613-21.

18. Metnitz PGH, Krenn CG, Steltzer H, et al. Effect of acute renal failure requiring renal replacement therapy on outcome in critically ill patients. Crit Care Med. 2002;30:2051-58.

19. Metcalfe W, Simpson $M$, Khan IH, et al. Acute renal failure requiring renal replacement therapy: incidence and outcome. QJM. 2002;95:579-83.

20. Legrand M, Bezemer R, Kandil A, et al. The role of renal hypoperfusion in development of renal microcirculatory dysfunction in endotoxemic rats. Intensive Care Med. 2011;37(7):1534-42.

21. Evans RG, Ince C, Joles JA, et al. Haemodynamic influences on kidney oxygenation: clinical implications of integrative physiology. Clin Exp Pharmacol Physiol. 2013;40(1):106-22.

22. Badr KF, Ichikawa I. Prerenal failure: a deleterious shift from renal compensation to decompensation. N Engl J Med. 1988;319:623-29.

23. Legrand M, Payen D. Understanding urine output in critically ill patients. Ann Intensiva Care. 2011;1(1):13.

24. Mitrou A, Gupples NG. WA. Renal blood flow dynamics in inbred rat strains provides insight into autoregulation. Curr Vasc Pharmacl. 2014;12(6):801-9.

25. Lerolle N, Guérot E, Faisy C, Bornstain C, Diehl JL, Fagon JY. Renal failure in septic shock: Predictive value of Doppler-based renal arterial resistive index. Intensive Care Med. 2006;32:1553-9.

26. Blomley MJ, Albrecht T, Cosgrove DO, et al. Liver vascular transit time analyzed with dynamic hepatic venography with bolus injections of an US contrast agent: early experience in seven patients with metastases. Radiology. 1998;209:862-6.

27. Cole L, Bellomo R, Silvester W, Reeves JH. A prospective, multicenter study of the epidemiology, management, and outcome of severe acute renal failure in "closed" ICU system. Am J Respir Crit Care Med. 2000;162:191-96.

28. Hoste EA, Lameire NH, Vanholder RC, Benoit DD, Decruyenaere JM, Colardyn FA. Acute renal failure in patients with sepsis in a surgical ICU: predictive factors, incidence, comorbidity, and outcome. J Am 
Soc Nephrol. 2003;14:1022-30.

29. Nourbakhsh N, Singh P. Role of renal oxygenation and mitochondrial function in the pathophysiology of acute kidney injury. Nephron Clin Pract. 2014;127(1-4):149-52.

30. Li W, Qiu X, Jiang H, et al. Ulinastatin inhibits the inflammation of LPS-induced acute lung injury in mice via regulation of AMPK/ NF-KB pathway. Int Immunopharmacol. 2015;29:560-7.

\section{Tables}

Table 1: Pathologic change for a semi-quantitative evaluation of kidney injury

\begin{tabular}{ll}
\hline Grade & Pathologic changes under light microscope \\
\hline 0, & Renal histopathology. \\
1, Mild & $\begin{array}{l}\text { Mild interstitial edema, renal tubular necrosis, slight glomerular capillaries angiectasis, and } \\
\text { inflammatory cells infiltration. }\end{array}$ \\
2, & Pathologic changes of renal between mild and severe. \\
Moderate & \\
3, Severe & $\begin{array}{l}\text { Severe interstitial edema, renal tubular necrosis, severe glomerular capillaries angiectasis, } \\
\end{array}$ \\
& mesangial proliferation, and severe inflammatory cells infiltration. \\
\hline
\end{tabular}

Table 2 Histopathology outcomes

\begin{tabular}{lllllll}
\hline & SH & SS & VAN & UTI & F & P \\
\hline Lung injury scores & $0.20 \pm 0.45$ & $4.00 \pm 0.87$ & $4.00 \pm 0.87$ & $2.78 \pm 0.83$ & 29.247 & $<0.001$ \\
& $* * \#$ \$ & & & $* * \# \#$ & & \\
TUNEL (\%) & $1.50 \pm 0.23$ & $32.22 \pm 1.71$ & $30.11 \pm 2.67$ & $13.78 \pm 2.91$ & 266.872 & $<0.001$ \\
& $* * \# \# \$$ & & & $* * \# \#$ & & \\
\hline
\end{tabular}

SH: the sham group; SS: the septic shock group; VAN: the vancomycin group; UTI: the ulinastatin group. * $\mathrm{P}<0.05$ vs. the SS group; ** $\mathrm{P}<0.01$ vs. the SS group; \# $\mathrm{P}<0.05$ vs. the VAN group; \#\# $\mathrm{P}<0.01$ vs. the VAN group; $\$ \mathrm{P}<0.05$ vs. the UTI group; $\$ \mathrm{P}<0.01$ vs. the UTI group.

\section{Figures}


Figure 1

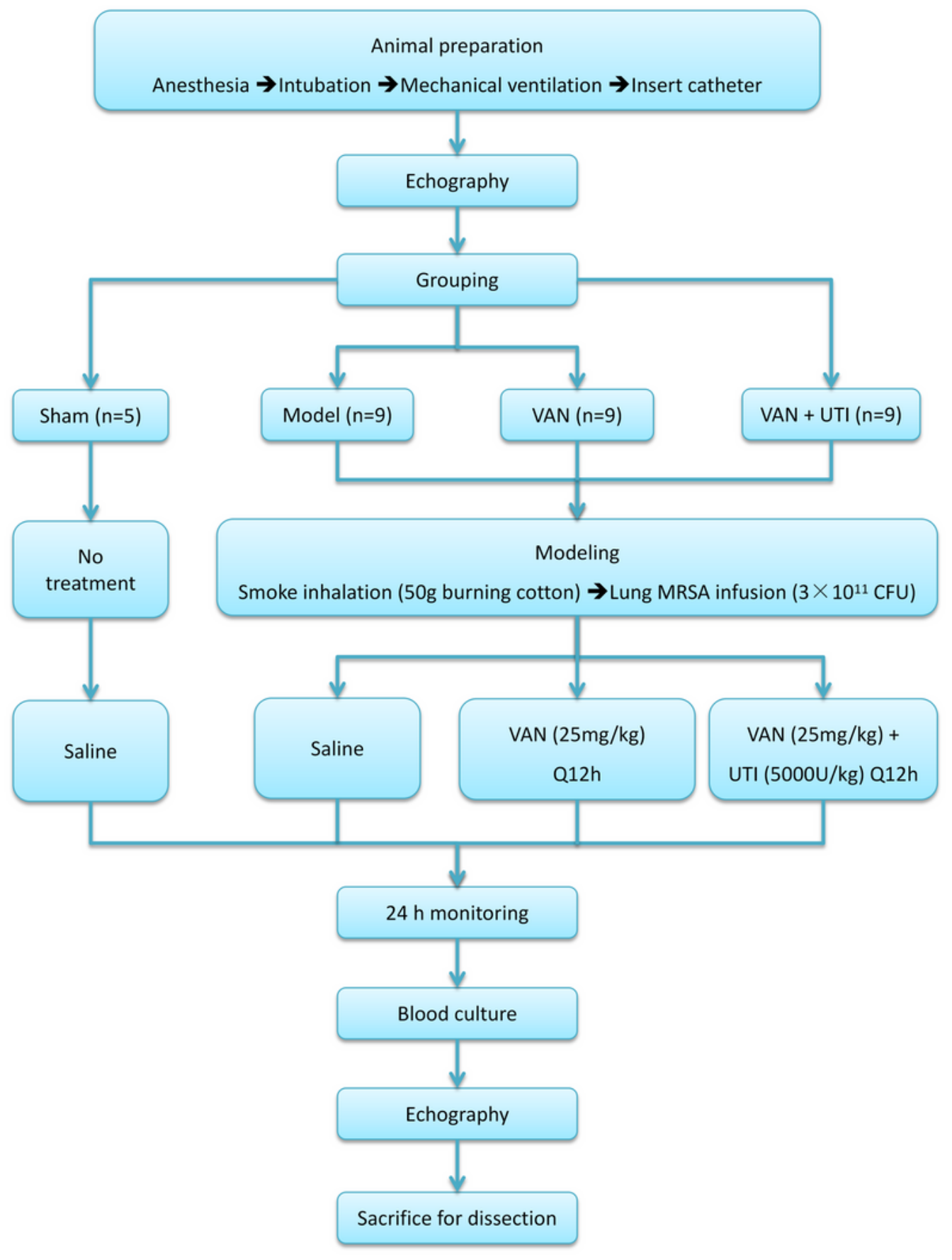

\section{Figure 1}

The diagram of the protocol. UTI: ulinastatin; VAN: vancomycin; MRSA: methicillin-resistant Staphylococcus aureus. 
Figure 2

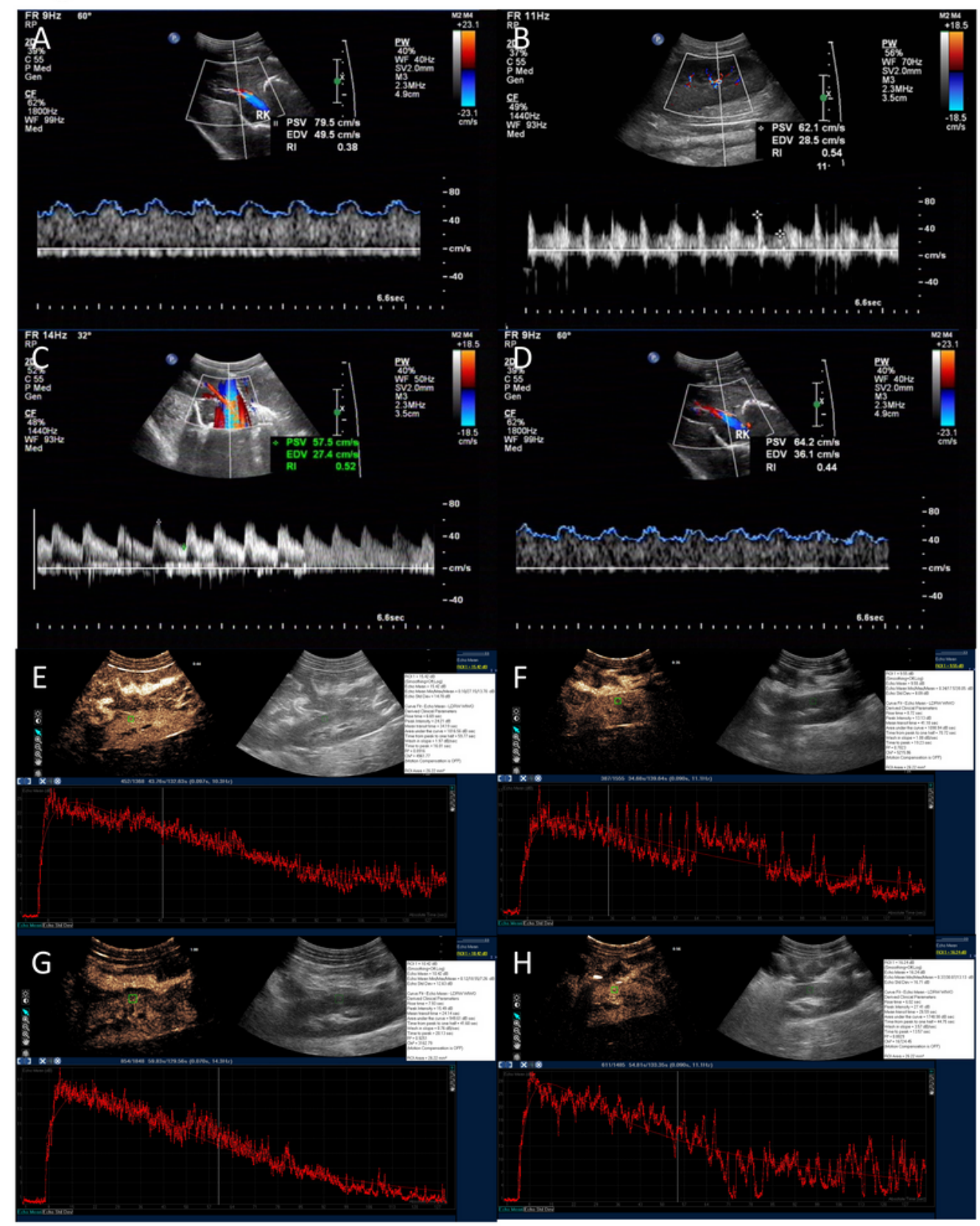

\section{Figure 2}

The Doppler gain was set in order to obtain a clear outline of flow waves with minimal background noise. An interlobar or arcuate artery was selected and the results were shown automatically on the screen. A: the SH group; B: the SS group; C: the VAN group; D: the UTI group. For CEUS, $2 \mathrm{ml}$ of sulphur hexafluoride microbubbles (8ul/ml) (SonoVue ${ }^{\circledR}$, Bracco Suisse SA, Plan-Les-Ouates, Switzerland) was bolus injected via the central venous catheter. A ROI ( $26 \mathrm{~mm} 2$, green square) was manually drawn within the renal cortex 
to create a TIC with the ultrasound system's QLAB software (red curves). The Pi, AUC, Th, Tp and Slope were calculated by the software automatically. E: the SH group; F: the SS group; G: the VAN group; H: the UTI group. SH: the sham group; SS: the septic shock group; VAN: the vancomycin group; UTI: the ulinastatin group; CEUS: contrast enhanced ultrasound; ROI: region of interest; TIC: time-intensity curve; Pi: peak intensity; AUC: area under curve; Th: time from peak to one half; Tp: time to peak; Slope: wash in slope.

Figure 3
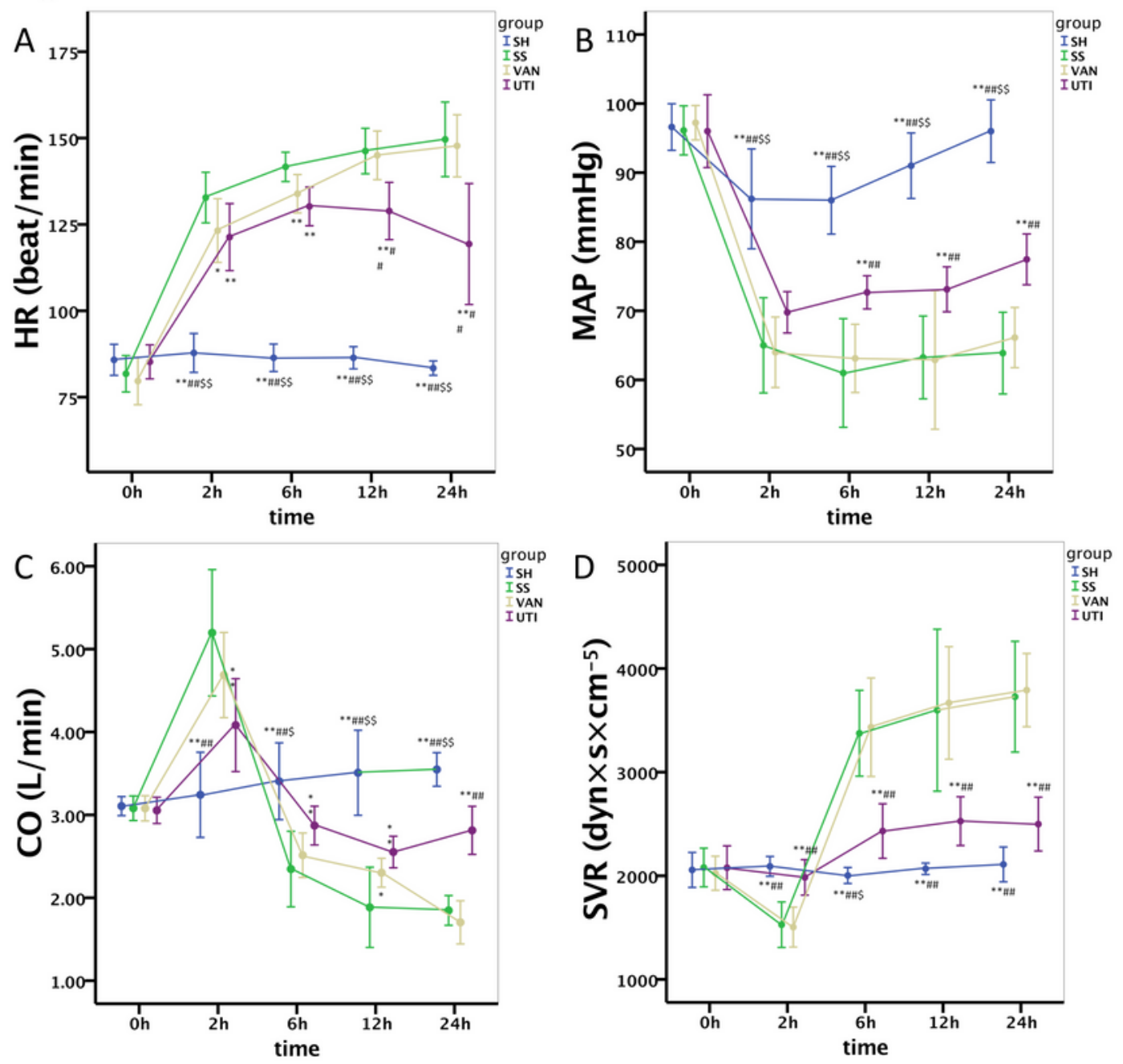

Figure 3 
All parameters in the $\mathrm{SH}$ group stayed at a relatively normal level throughout the entire protocol. The changes in the VAN group were similar with the SS group. A: In the UTI group, HR decreased slowly after $12 \mathrm{~h}$ post-injury. B: In the SS and VAN group, MAP decreased to $60-70 \mathrm{mmHg}$ at $2 \mathrm{~h}$ post-injury, and stayed at a low level. In the UTI group, MAP was improved progressively after $6 \mathrm{~h}$ post-injury. C, D: The changes of CO and SVR in the SS and VAN group were in opposite direction: the CO increased at $2 \mathrm{~h}$ postinjury and then gradually decreased, whereas the SVR decreased at the same time point and then gradually increased. The fluctuation of $\mathrm{CO}$ in the UTI group was ameliorated. MAP: mean arterial pressure; CO: cardiac output; SVR: systemic vascular resistance. SH: the sham group; SS: the septic shock group; VAN: the vancomycin group; UTI: the ulinastatin group. * $\mathrm{P}<0.05$ vs. the SS group; ${ }^{* \star} \mathrm{P}<0.01$ vs. the SS group; \# $\mathrm{P}<0.05$ vs. the VAN group; \#\# P<0.01 vs. the VAN group; $\$ \mathrm{P}<0.05$ vs. the UTI group; $\$$ $P<0.01$ vs. the UTI group. 
Figure 4
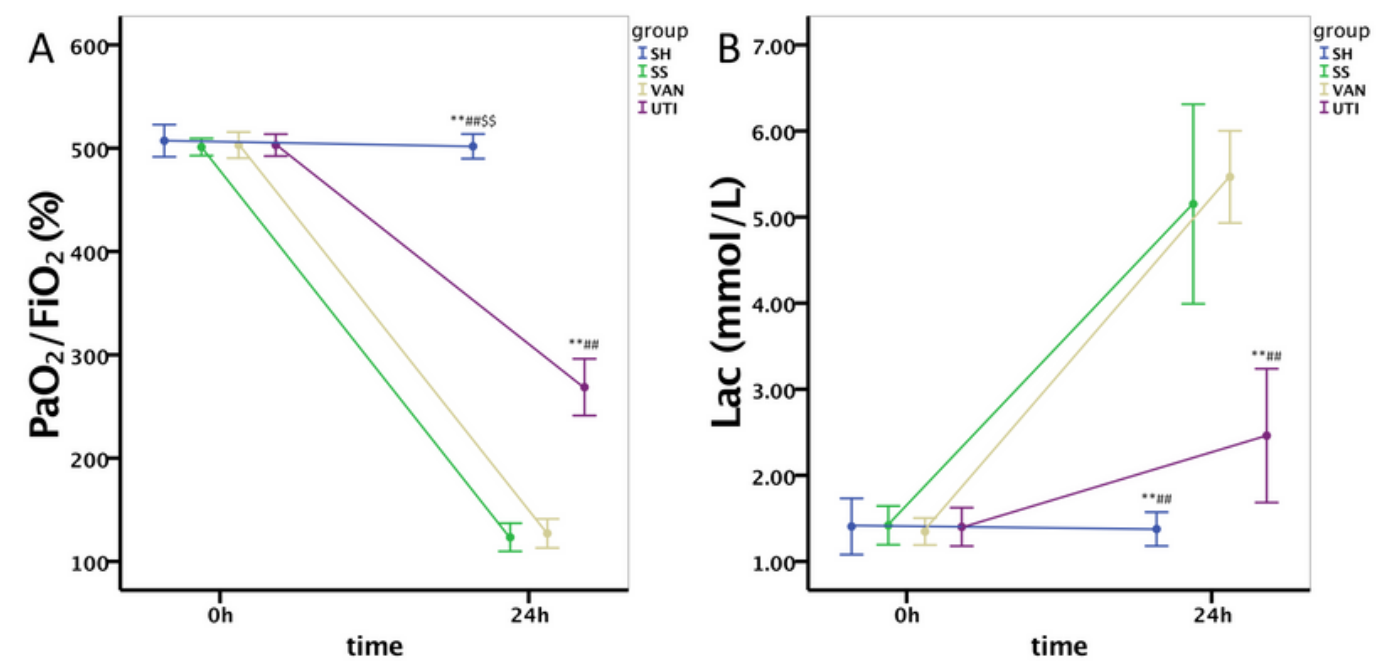

\section{Figure 4}

A: At the end of the protocol, $\mathrm{PaO} 2 / \mathrm{FiO} 2$ in the UTI group was higher than the former tow groups $(\mathrm{P}<$ 0.001), even was lower than the SH group. B: Same with the Lac. Lac: lactic acid. SH: the sham group; SS: the septic shock group; VAN: the vancomycin group; UTI: the ulinastatin group. * $P<0.05$ vs. the SS group; ${ }^{*} \mathrm{P}<0.01$ vs. the SS group; \# $\mathrm{P}<0.05$ vs. the VAN group; \#\# $\mathrm{P}<0.01$ vs. the VAN group; $\$ \mathrm{P}<0.05$ vs. the UTI group; $\$ \mathrm{P}<0.01$ vs. the UTI group. 
Figure 5
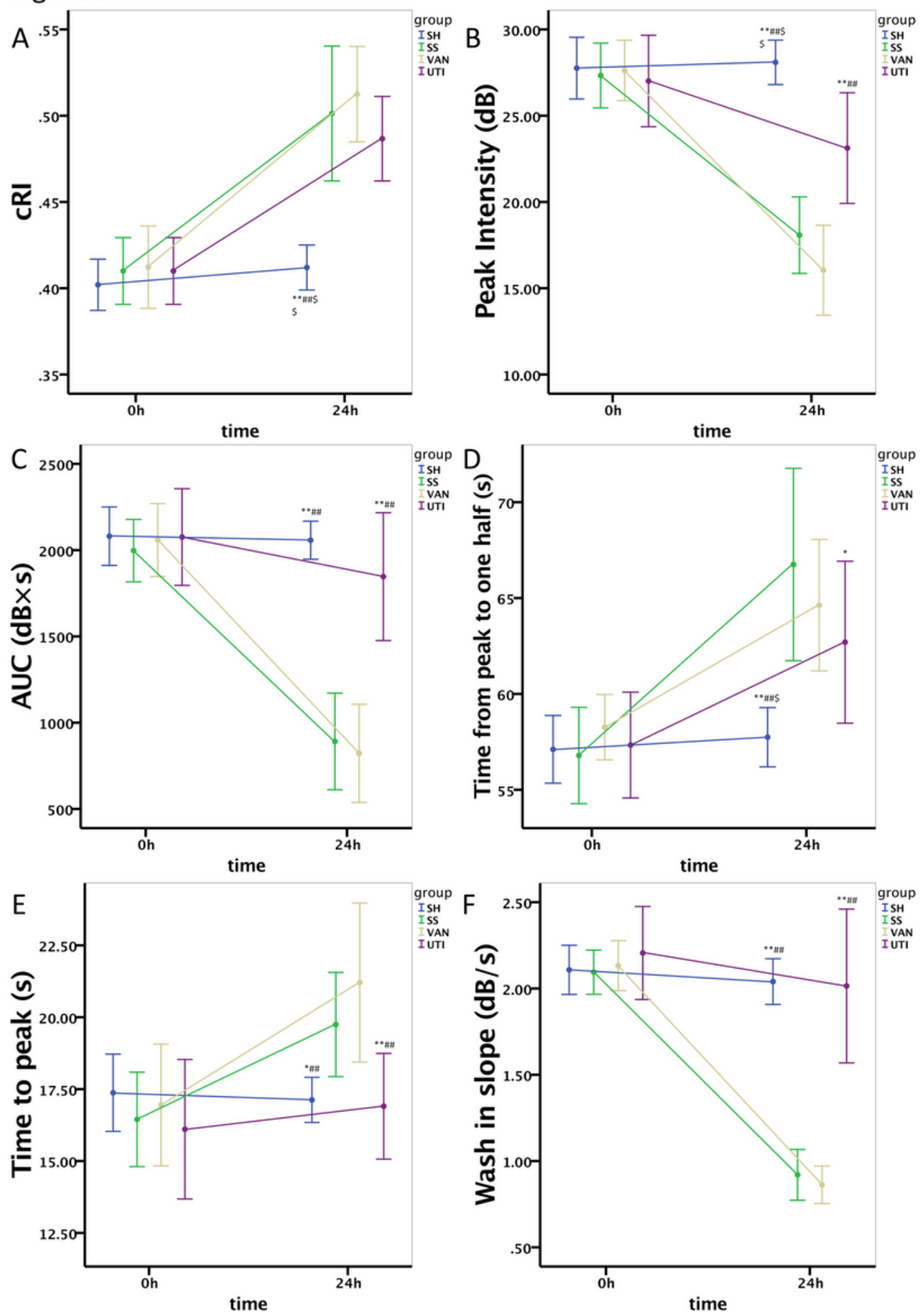

Figure 5

A: $\mathrm{CRI}$ increased significantly at the end of the protocol except the SH groups.B: Peak intensity decreased significantly after injury in all experimental groups. The improvement was found in the UTI group than the SS and VAN group. C: AUC decreased post-injury in the SS and VAN group, but was improved in the UTI group. D, E: The time from peak to one half and the time to peak significantly increased in the SS and VAN group whereas improved by the UTI treatment. F: Wash in slope significantly decreased in the SS 
and VAN group whereas improved by the UTI treatment. cRI: corrected resistive index; AUC: area under curve. SH: the sham group; SS: the septic shock group; VAN: the vancomycin group; UTI: the ulinastatin group. * $P<0.05$ vs. the SS group; ${ }^{* *} P<0.01$ vs. the SS group; \# $P<0.05$ vs. the VAN group; \#\# $P<0.01$ vs. the VAN group; $\$ P<0.05$ vs. the UTI group; $\$ P<0.01$ vs. the UTI group

Figure 6

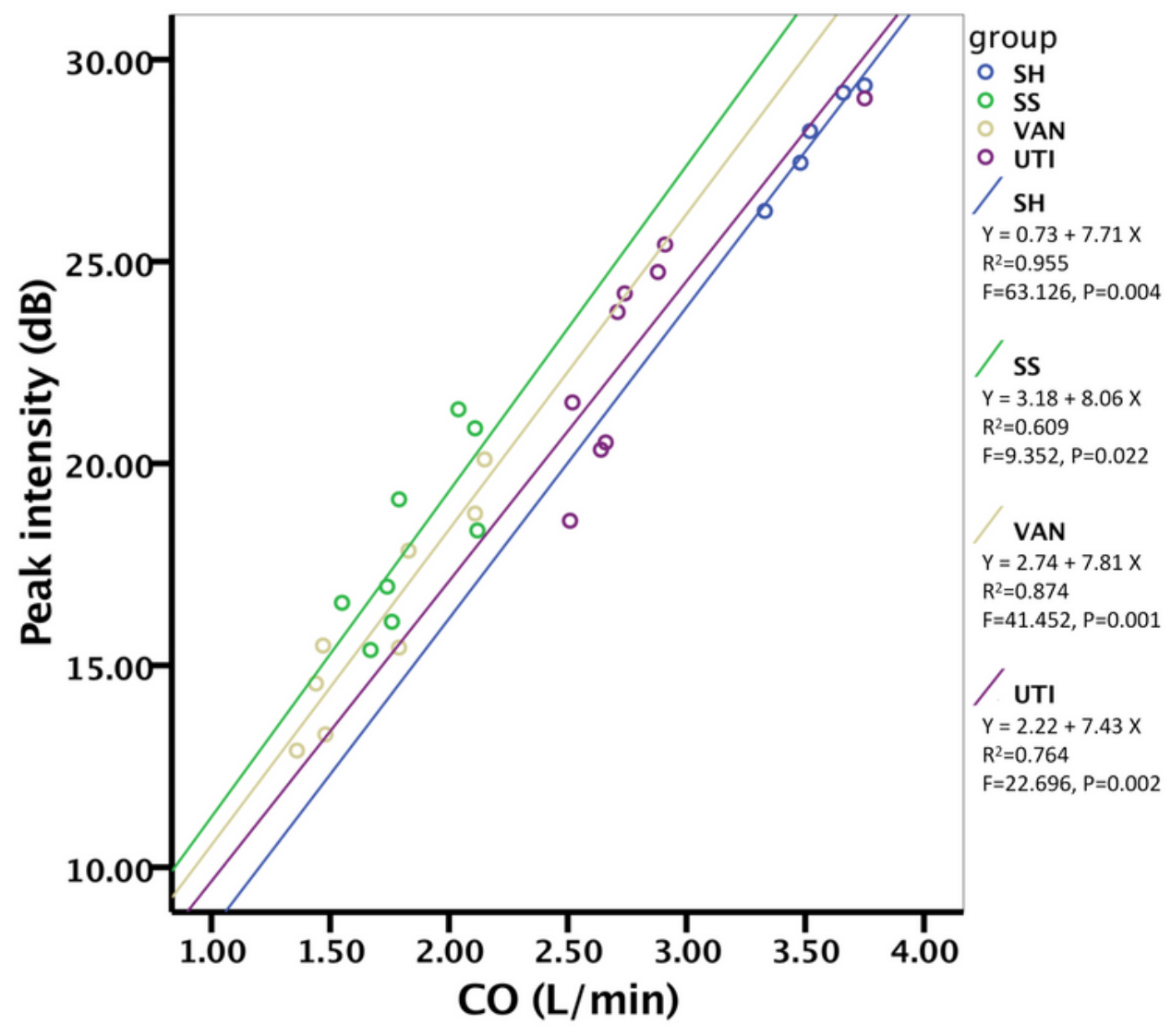

Figure 6 
Linear regression was found between the $\mathrm{CO}$ and peak intensity in each group. There were similar regression coefficients in all groups. CO: cardiac output. SH: the sham group; SS: the septic shock group; VAN: the vancomycin group; UTI: the ulinastatin group.

\section{Figure 7}

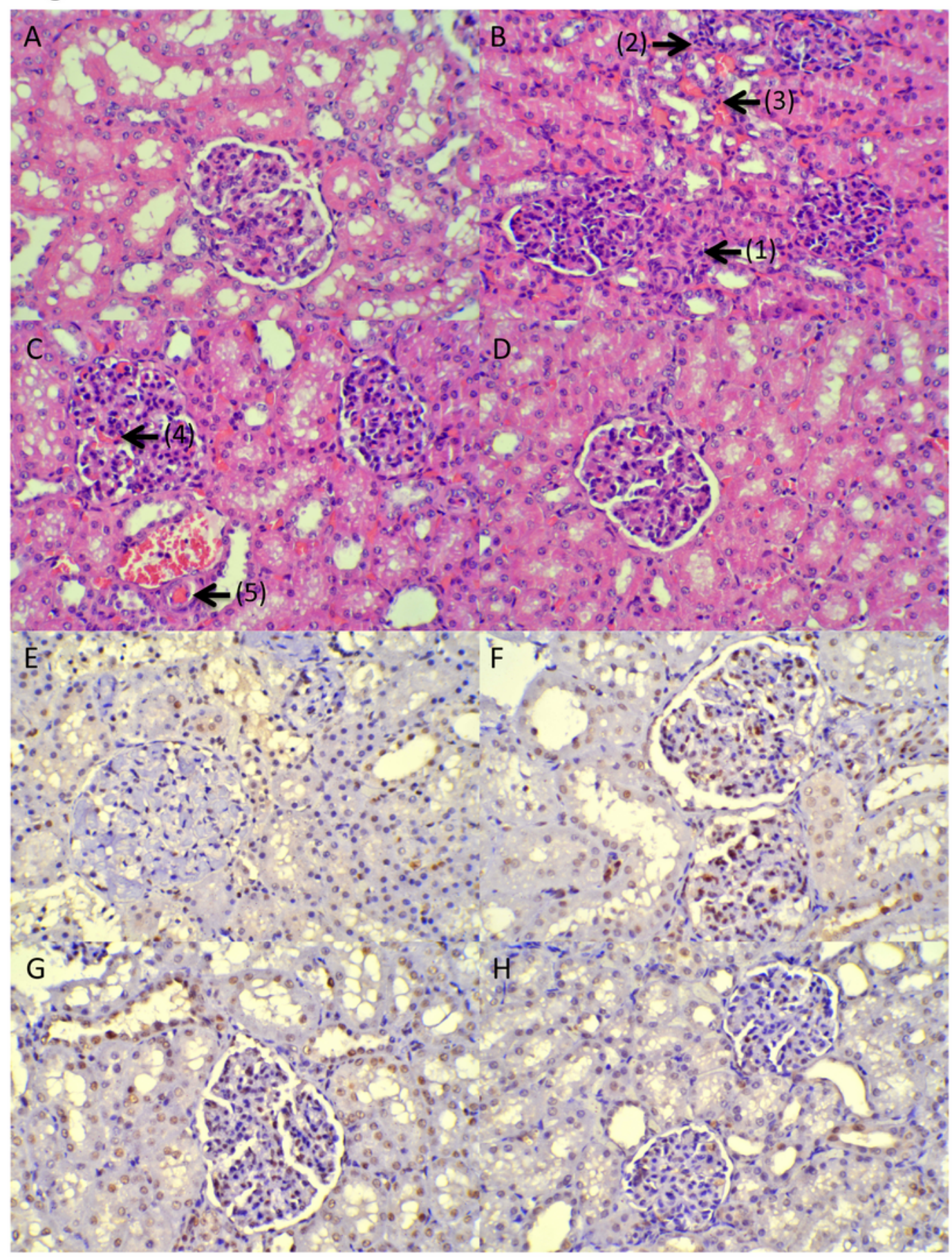

\section{Figure 7}

Under the light microscope, the renal injury was prominent in all experimental groups $(\times 400)$. (1) fibroblasts proliferation in the renal mesenchyme beside the glomerulus; (2) inflammatory cells 
infiltration; (3) renal interstitial hyperemia; (4) glomerular capillaries angiectasis and hyperemia; (5) microthrombus formation. A: the sham (SH) group; B: the septic shock (SS) group; C: the vancomycin (VAN) group; D: the ulinastatin (UTI) group. TUNEL assay revealed that there were greater numbers of apoptotic cells in the SS and VAN group than the sham and UTI group. Cells that suffered with apoptotic signaling cascades were marked with brown. E: the SH group; F: the SS group; G: the VAN group; H: the UTI group.

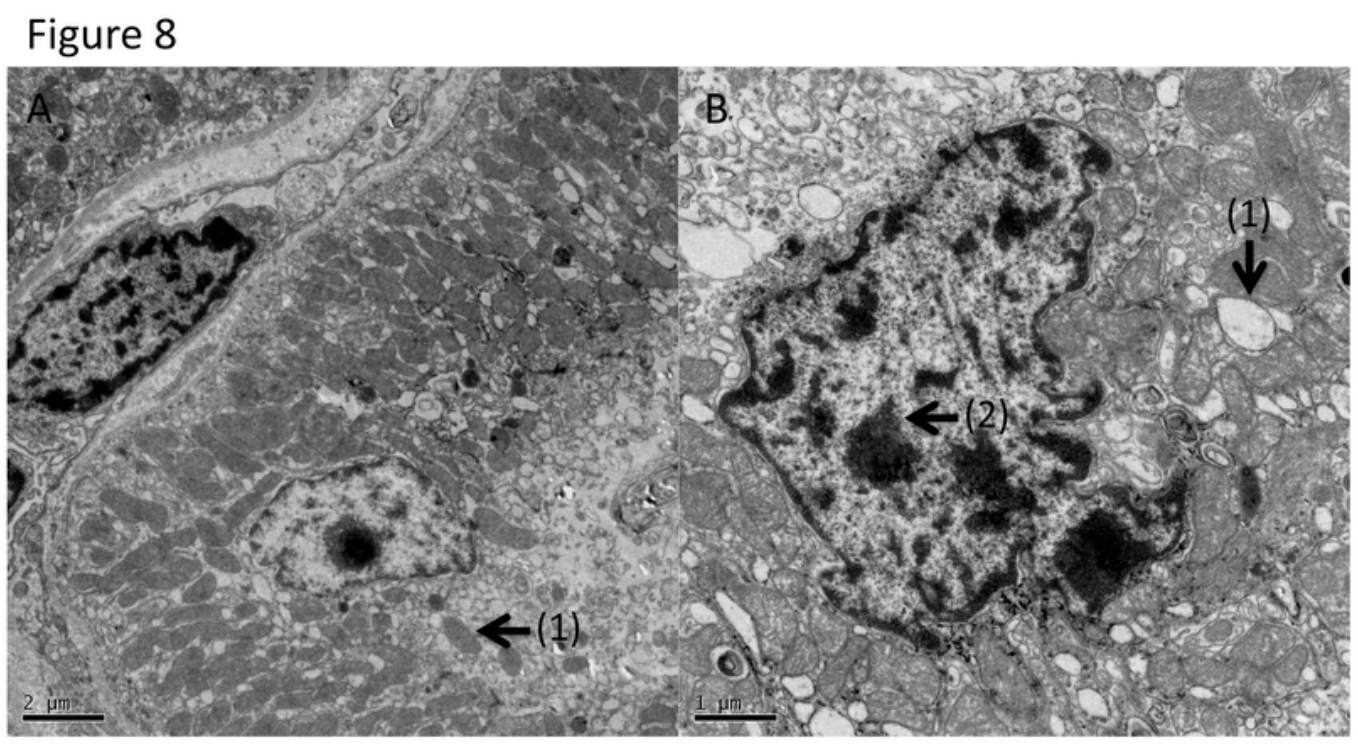

\section{Figure 8}


The images of transmission electron microscope from the septic shock group. (1) the mitochondria swelled and the cristae disappeared; (2) the chromatin and nucleus vacuolar of epithelium were degenerated

\section{Supplementary Files}

This is a list of supplementary files associated with this preprint. Click to download.

- model.mp4

- sham.mp4

- uti.mp4

- van.mp4 